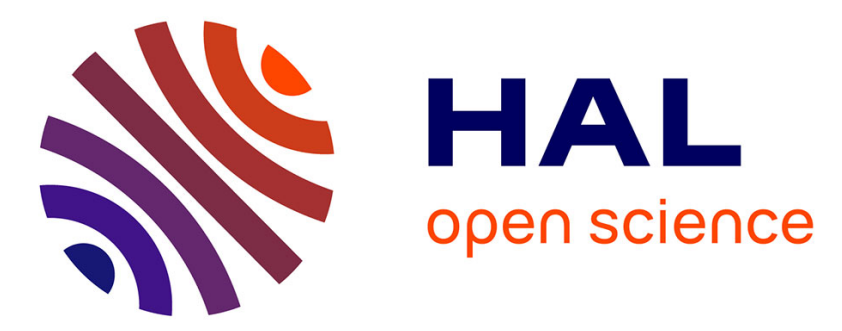

\title{
MaxEnt feature-based reliability model method for real-time detection of early chatter in high-speed milling
}

Yanqing Zhao, Kondo Hloindo Adjallah, Alexandre Sava, Zouhang Wang

\section{To cite this version:}

Yanqing Zhao, Kondo Hloindo Adjallah, Alexandre Sava, Zouhang Wang. MaxEnt feature-based reliability model method for real-time detection of early chatter in high-speed milling. ISA Transactions, 2021, 113 (2021), pp.39-51. 10.1016/j.isatra.2020.07.022 . hal-03018329

\section{HAL Id: hal-03018329 \\ https://hal.univ-lorraine.fr/hal-03018329}

Submitted on 4 Dec 2020

HAL is a multi-disciplinary open access archive for the deposit and dissemination of scientific research documents, whether they are published or not. The documents may come from teaching and research institutions in France or abroad, or from public or private research centers.
L'archive ouverte pluridisciplinaire HAL, est destinée au dépôt et à la diffusion de documents scientifiques de niveau recherche, publiés ou non, émanant des établissements d'enseignement et de recherche français ou étrangers, des laboratoires publics ou privés.

\section{(ㅇ)(1) $\$$}

Distributed under a Creative Commons Attribution - NonCommercial - NoDerivatives 44.0 


\title{
MaxEnt Feature-based Reliability Model Method for Real-time Detection of Early Chatter in High-Speed Milling
}

\author{
Yanqing Zhao a,b, Kondo H. Adjallah ${ }^{\mathrm{a}, *}$, Alexandre Sava ${ }^{\mathrm{a}}$, Zhouhang Wang ${ }^{\mathrm{a}}$ \\ ${ }^{a}$ Université de Lorraine, LCOMS, F-57000 Metz, France \\ ${ }^{\mathrm{b} J i a n g s u}$ Key Laboratory of Traffic and Transportation Security, Huaiyin Institute of \\ Technology, Huai' an, 223003, China \\ *Corresponding author Email: kondo.adjallah@univ-lorraine.fr
}




\title{
MaxEnt Feature-based Reliability Model Method for Real-time Detection of Early Chatter in High-Speed Milling
}

\begin{abstract}
Real-time detection of early chatter is a vital strategy to improve machining quality and material removal rate in the high-speed milling processes. This paper proposes a maximum entropy (MaxEnt) feature-based reliability model method for real-time detection of early chatter based on multiple sampling per revolution (MSPR) technique and second-order reliability method (SORM). To enhance the detection reliability, the MSPR is used to acquire multiple sets of once-per-revolution sampled data (i.e., MSPR data) and to overcome the shortcoming of the once-per-revolution sampling. The proposed MaxEnt feature-based reliability model method solves the issue of the real-time detection of early chatter while ensuring its reliability. The failure hazard function (FHF) is estimated as a chatter indicator by using the SORM with the MaxEnt feature. The proposed method consists of five steps. First, set the prior parameters. Then collect data by using the MSPR technique. Next, calculate a set of the standard deviation of the data collected as a chatter feature and estimate the chatter indicator FHF by applying the SORM with the MaxEnt feature. Finally, implement the real-time detection of early chatter based on the estimated chatter indicator FHF and the threshold $\mathrm{FHF}_{0}$. The proposed method is applied to the high-speed milling process. Two examples prove that the proposed method can detect two kinds of early chatter: the early-stage of a severe chatter and the slightly intolerable chatter.
\end{abstract}

Keywords: Early chatter detection, high-speed milling, maximum entropy, reliability method, sampling frequency.

\section{Introduction}

In the modern aerospace and automotive industry, the high-speed milling machine has been widely employed to improve material removal rate and to obtain high-quality machining surface. In the high-speed milling process, one of the most common phenomena to be handled is the chatter, which is a self-excited 
vibration phenomenon causing instability in the cutting process [1]. The appearance of the chatter reduces productivity, accelerates wear of the cutting tool, and leads to poor machining quality. The early chatter should be detected as soon as possible to avoid the damage of the work-piece and save time in the chatter control loop [2].

The chatter can be detected by identifying the chatter feature and monitoring the transition between the chatter-free and chatter stages. As a general scheme of fault detection, the procedure of early chatter detection also consists of three steps: data acquisition, feature extraction, and decision-making. Among them, the feature extraction is the most important for early chatter detection. Therefore, significant research works have been proposed to extract reliable chatter features based on signal processing methods and mathematical models of chatter physics.

The chatter signal during machining has complex nonlinear and non-stationary characteristics. So, authors employed nonlinear and non-stationary signal processing methods in the time domain [3][4], frequency domain [5], and time-frequency domain [6][7][8] to detect the early chatter.

In the time domain, the chatter feature can be extracted by calculating the statistical characteristics of the collected signal, such as mean, variance, and standard deviation [3]. Jia et al. [4] presented a synthetic criterion (SC) by integrating standard deviation and one-step auto-correlation function for early chatter detection. The experimental results verified that SC can detect the early chatter. However, the variation of machining parameters deteriorates the chatter detection performance of the SC.

In the frequency domain, based on the energy aggregation property of the chatter, Hynynen et al. [5] proposed to use the coherence function of the acceleration signal and the audio signal to detect the early chatter. The experimental results showed that the proposed method is sensitive to the early chatter. Nevertheless, the proposed method is not independent of machining parameters, so the optimal threshold must be updated with the variation of machining parameters. As to this problem, Aslan et al. [9] and Cao et al. [3] have explored the independent chatter detection methods and suggested using the comb filter. Due 
to employing this filter, the tooth passing frequency, spindle rotation frequency, and corresponding harmonics are filtered from the chatter signal. Thus, the chatter feature based on the filtered chatter signal is independent of machining parameters. However, it is a challenge to determine the number of spaced notches of the comb filter [10]. This disadvantage limits the application of comb filter-based methods in early chatter detection.

In the time-frequency domain, wavelet transform (WT), empirical mode decomposition (EMD), and variational mode decomposition (VMD) are standard signal processing methods to detect an early chatter. These advanced signal processing methods decompose the chatter signal into some components/modes corresponding to different frequency bands. The decomposition components/modes, including rich chatter information, can serve to highlight and extract the chatter features. However, WT-based and VMD-based chatter detection methods need to determine optimal decomposition parameters in advance. In practice, an unsuitable choice of decomposition parameters affects the detection effectiveness. Up to now, there is no selection criterion of the optimal decomposition parameters. Although EMD-based chatter detection methods do not need to determine optimal decomposition parameters in advance, their applications are limited, due to no strengthened theoretical basis [11].

The abovementioned chatter detection methods rely on signal processing and do not consider the mathematical model of chatter physics. Some chatter detection methods using the mathematical model of chatter physics were also introduced for real-time implementation. The mathematical model of chatter physics can be categorized into process signal model [12][13], process state model [14][15], bifurcation and chaos model [16][17][18], and probabilistic process model [19][20][21].

Some researchers adopted the nonlinear process to model the milling process time-varying dynamics due to the milling process signal nonlinear characteristics. The autoregressive moving average (ARMA) model and exponential Autoregressive (ExpAr) model are typical. Van Dijk et al. [12] proposed a parametric modeling approach for detecting the chatter in the milling process. The parametric modeling approach utilizes the ARMA model of the signal. The proposed model-based method can detect chatter faster in a 
high-speed milling process. However, it gives no solution to determine the order of the ARMA model. The estimation results may be unsatisfactory if the chosen order of the ARMA model is not suitable. Similarly, the inappropriate parameters of the ExpAr model can also lead to disappointing results [13].

Dynamic state models, incorporating the milling process dynamic properties, were also employed to detect chatter. For instance, Kakinuma et al. [14] designed a disturbance observer based on the spindle current and speed signals to detect chatter. The chatter and forced vibrations are separated by using digital filters. They verified the effectiveness of the disturbance observer using several milling tests. However, there is no principle to set the frequency bands of the designed digital filters. Caliskan et al. [15] proposed a Kalman filter to detect and extract chatter components. The Kalman filter is assigned to estimate the forced vibration component. Then the chatter vibration component can be obtained by subtracting the forced vibration component from the measured signal. However, the measured signal is nonlinear and non-stationary. The chatter vibration component obtained using the Kalman filter may not depict the character of the chatter adequately, since the Kalman filter's ability is limited for processing nonlinear and non-stationary signals.

The chatter is an unstable phenomenon related to bifurcation or chaos. Thus, some researchers addressed the chatter detection problem based on the bifurcation and chaos model [22][23][24]. Schmitz et al. [16] used the once-per-revolution sampling technique to detect chatter. As the name suggests, the once-perrevolution sampling technique consists in collecting one sample in each spindle revolution synchronously. Based on the property of the milling signal with a constant revolution speed, one can find that the onceper-revolution sampled data is constant at the chatter-free stage. However, the once-per-revolution sampled data has fluctuations at the chatter stage. In theory, due to the different distributions of the once-perrevolution sampled data at chatter-free and chatter stages, the chatter can be detected easily [16][17][18]. The once-per-revolution sampled data is independent of the machining parameters and can be used to detect chatter

In practice, the measured signal includes noise, due to some uncontrollable influences, such as the measurement instrument and in-homogeneous material of the work-piece [25]. In this situation, the 
boundary between chatter-free and early chatter is fuzzy. We considered this problem in a previous work where we proposed a cumulative early chatter detection method based on the once-per-revolution sampling technique and sequential probability ratio test [26]. The early chatter was detected through accumulating the unobvious chatter feature.

However, a set of once-per-revolution sampled data cannot depict the chatter character adequately because each set of once-per-revolution sampled data only depicts partial chatter information [27]. For the quasiperiodic chatter associated with the Hopf bifurcation, each set of once-per-revolution sampled data has fluctuation which can be used to depict the appearance of the chatter. However, for the period-2 chatter associated with the period-2 bifurcation, there is a set of once-per-revolution sampled data without the fluctuation [27]. The chatter-free signal and the period-2 chatter cannot be distinguished based on this set of once-per-revolution sampled data. Thus, the early chatter detection methods based on the once-perrevolution sampling technique need further development for practical applications.

Indeed, to acquire the information of the high order harmonics, the sampling frequency is set much higher than the spindle rotation frequency. Thus, in one spindle revolution, several samples can be collected. In this situation, some sets of once-per-revolution sampled data can be collected by implementing the onceper-revolution sampling repeatedly. With this in mind, this paper presents a multiple sampling per revolution (MSPR) and shows its performance. The idea of MSPR is to conduct the once-per-revolution sampling repeatedly at different sampling instants. Thus, MSPR data, i.e., multiple sets of once-perrevolution sampled data, can be obtained and used for early chatter detection. Generally, the larger the number of data considered, the more information will be obtained, and the results will be more reliable. The MSPR overcomes the shortcoming of once-per-revolution sampling and can describe the chatter feature adequately. Thus, the exploration of the data resource enhances reliability in describing the chatter feature. Due to the independence of once-per-revolution sampled data of machining parameters, the MSPR data composed of multiple sets of once-per-revolution sampled data is also independent of machining parameters. Thus, the chatter detection methods based on MSPR are independent of machining parameters. 
In the milling process, many random factors affect the appearance of the chatter, such as degradation of the machine, uncertainties of machining parameters, and in-homogeneous material [20]. Therefore, the probabilistic process model is suitable for chatter prediction and detection [28].

In industrial engineering, reliability analysis is necessary to ensure the safety and serviceability of systems or parts. Referring to Rausand in [21], the chatter issue is related to the physic reliability of a work-piece. So, it appears relevant to use the physic reliability-based approach for preventing chatter related failure in a milling process. Such a failure can appear after chatter symptoms before a tool breaks or a product lose its quality during machining. Hence, we consider the physic reliability definition of a work-piece as a probability that the strength of such an item is higher than the load corresponding, in our case, to stresses imposed on it during operation. The illustration in [21] describes perfectly the physic reliability of a workpiece in a milling process, where tools, machine components, and work-piece quality could degrade when the strength of a component drops below the stresses or loads. The decrease of the strength may correspond to the tool wearing or the loss of mechanical properties of the work-piece material, or the degradation of the machine component. In reliability analysis, it is a challenge to focus on design aspect (or the most probable failure point). By applying the second-order reliability method (SORM) based on the maximum entropy (MaxEnt) feature, during machining, one can avoid focusing on design consideration. Also, the SORM based on the MaxEnt feature can estimate quickly the failure hazard function (FHF), which is used as a chatter indicator.

The MaxEnt principle, with the first four moments, is a mature method and has been widely used to estimate the probability density distribution (PDF) [29][30][31][32]. In the context of the moment methods, more moments represent more information about system behavior. However, higher-order moments may lead to the occurrence of instability in estimating the PDF as well. To deal with this problem, Rajan et al. proposed an improved MaxEnt method [33], which can estimate the PDF through accommodating higher-order moments. However, the computational cost is expensive when the MaxEnt principle accommodates higherorder moments. To improve the performance of the MaxEnt principle, researchers introduced the concept 
of the fractional moments to estimate the PDF [34][35]. Compared to the MaxEnt principle with integer moments, the MaxEnt principle with fractional moments have lower sampling variability and can optimize the set of moments. Nonetheless, there are several issues in the MaxEnt principle with fractional moments. First, its use is limited in some engineering applications, since the fractional moments are not valid for random variables that can take negative values. The second issue concerns with an optimization problem. Since the objective function is non-continuous and non-convex, it is difficult to obtain the optimization [36]. Therefore, we propose to use the MaxEnt feature with the first four moments to estimate the PDF in this work.

We proposed to detect early chatter based on both signal processing and feature extraction jointly with probabilistic process modeling.

This paper addresses two aspects of the early chatter detection: (1) to detect the early stage of the severe chatter quickly when it just appears and is not developed completely; (2) to detect the slightly intolerable chatter accurately. The detection of these two kinds of early chatter has significance in machining processes. On the one hand, before the complete development of severe chatter, there are no noticeable chatter marks left on the work-piece [12]. Thus, the implementation of the early detection of severe chatter can ensure surface quality and to increase the material removal rate.

On the other hand, at present, to increase the material removal rate, the machining parameters are set usually nearly the boundary between the stable and unstable regions. Under this machining condition, the slight variation of machining parameters may lead to the slight or severe chatter. For some particular parts, the high precision machining surface is necessary, and even slight chatter may be intolerable [37]. Thus, the reliable detection of the slightly intolerable chatter also has significance in the high precision process, especially for the valuable parts.

This work aims to design a method for detecting early chatted. For that purpose, we rely on the secondorder reliability model (SORM), which is a function of the chatter physics process, through the MaxEnt 
feature, and the MSPR. The MSPR allows for acquiring synchronous data, which are independent of machining parameters. The proposed chatter detection method is also independent of machining parameters. Also, the proposed method avoids focusing on design considerations by using the MaxEnt feature-based SORM. Thus the main contributions of this work are summarized as follows:

(I) The multiple sampling per revolution (MSPR) technique is proposed to collect MSPR data for overcoming the shortcoming of the once-per-revolution sampling.

(II) An indicator, called failure hazard function (FHF), is introduced to indicate the early chatter reliably.

(III) A MaxEnt feature-based reliability model method, MFRM method for short, is proposed to detect the early chatter reliably and quickly.

We organize the rest of the paper as follows. The introduction of frequencies during the early chatter is given in Section 2. Section 3 illustrates the MSPR technique. The SORM based on the MaxEnt feature is introduced in Section 4. Section 5 shows the proposed MFRM method of real-time detection of early chatter. In Section 6, two examples validate the effectiveness of the proposed method. Finally, Section 7 lays out the final remarks and some suggestions for further research directions.

\section{Frequencies during early chatter}

The early chatter signal in a milling process includes multiple frequencies [38]. In the milling process, the cutting behavior is interrupted and different from the turning process. Therefore, the chatter signal of the milling process is different from the one produced in the turning process. The chatter signal of the milling process includes multiple chatter frequencies rather than a single chatter frequency. These multiple chatter frequencies show different phenomena of bifurcation or chaos that result, in general, in slight and severe chatter, respectively [39].

In practice, the bifurcation is the secondary Hopf bifurcation and Period-N bifurcation commonly [40]. According to the reference [38], for the secondary Hopf bifurcation, the frequencies arising in the early 
chatter signal are:

$$
f_{H}= \pm \frac{\omega}{2 \pi}+n \frac{s \Omega}{60}, n=\ldots,-1,0,1, \ldots
$$

where $\Omega$ represents spindle revolution speed and is expressed in revolution per minute, and $s$ is the tooth number of the cutting tool. The index $f_{H}$ represents frequencies of the secondary Hopf bifurcation. The chatter signal includes an infinite number of frequencies in theory. In practice, negative values $f_{H}$ have no physical meaning, and only positive values $f_{H}$ are detectable.

For the case of the period-N bifurcation, the frequencies $f_{P}$ during early chatter can be expressed as follows:

$$
f_{P}=\frac{s \Omega}{30(N-1)}+n \frac{s \Omega}{60(N-1)}, n=\ldots,-1,0,1, \ldots, N=2,3, \ldots
$$

where $N$ is a number corresponding to the kinds of the period-N bifurcation.

Except for the chatter frequencies, the early chatter signal also includes the typical frequencies $f_{N}$ which appear in the chatter-free process. The typical frequencies are produced by the normal cutting process and can be written in the simple form of

$$
f_{N}=\frac{n s \Omega}{60}, n=1,23, \ldots
$$

During the chatter-free process, there are only typical frequencies in the signal. The period of the signal is $\frac{60}{s \Omega}$. Therefore, the sampling data obtained by using the once-per-revolution sampling technique are constant. However, for the early chatter process, the chatter frequencies $\left(f_{H}\right.$ or $\left.f_{P}\right)$ arise in the chatter signal. Due to the appearance of the chatter frequencies, fluctuations can be observed in the sampling data obtained by using the MSPR technique. 


\section{Multiple sampling per revolution}

This section describes the concept of the MSPR, which is an insight into the synchronous sampling technique. First, we recall the principle of once-per-revolution sampling. According to the reference [17], the once-per-revolution sampling means to collect one sample in each revolution synchronously. In contrast, the multiple-per-revolution sampling (MSPR) collects multiple samples in one revolution synchronously through conducting the once-per-revolution sampling repeatedly.

It should be noted that the proposed MSPR is different from the traditional synchronous sampling used for order tracking [41]. The traditional synchronous sampling regards the sampled data as a series of synchronous data. However, the MSPR regards the sampled data as multiple sets of once-per-revolution sampled data.

Assuming there is a digital signal in the phase domain

$$
X=\left\{x\left(\phi_{0}\right), x\left(\phi_{0}+\frac{2 \pi}{M}\right), x\left(\phi_{0}+2 \frac{2 \pi}{M}\right), \ldots, x\left(\phi_{0}+(M-1) k \frac{2 \pi}{M}\right)\right\},\left(M, k \in N^{+}\right),
$$

where $\phi_{0}$ represents the initial sampling phase, $M$ is the sample number in one revolution, $\frac{2 \pi}{M}$ represents sampling interval, $k$ represents the number of revolutions.

According to the Nyquist-Shannon sampling theorem, the sampling frequency should be greater than twice the highest frequency of the signal. The sampling frequency is generally far higher than the rotation frequency to collect the information, including some high-order harmonics. So, it is possible to collect more samples in each revolution synchronously. The MSPR can be described as follows:

(1) At the initial sampling phase $\phi_{0}$, use the once-per-revolution sampling to collect the signal $X$, which yields a set $\left\{x\left(\phi_{0}\right), x\left(\phi_{0}+2 \pi\right), x\left(\phi_{0}+4 \pi\right), \ldots, x\left(\phi_{0}+2 k \pi\right)\right\}$ of once-per-revolution sampled data.

(2) At the sampling phases $\phi_{0}+m \frac{2 \pi}{M}(m=1,2,3,4, \cdots, M-1)$, collect the signal $X$ by using the once- 
per-revolution sampling repeatedly, then $M-1$ sets of once-per-revolution sampled data are collected.

After the $M$ implementations of the once-per-revolution sampling technique, we obtain $M$ sets of once-perrevolution sampled data. Due to multiple sampling in one revolution, this technique is called MSPR. However, for the traditional synchronous sampling used for order tracking, the synchronous sampled signal is the digital signal $X$. Thus, one can say that the MSPR brings an insight into the synchronous sampling technique. The MSPR data includes $M$ sets of once-per-revolution sampled data. In practice, the sample number $M$ should be set according to the applications. A large sample number $M$ allows obtaining more information and leads to a high sampling cost.

The proposed MSPR can overcome the shortcoming of the once-per-revolution sampling in the aspect of depicting the chatter character. For a displacement signal of the cutting tool, one can obtain once-perrevolution sampled data and MSPR data by using the once-per-revolution sampling and MSPR, respectively.

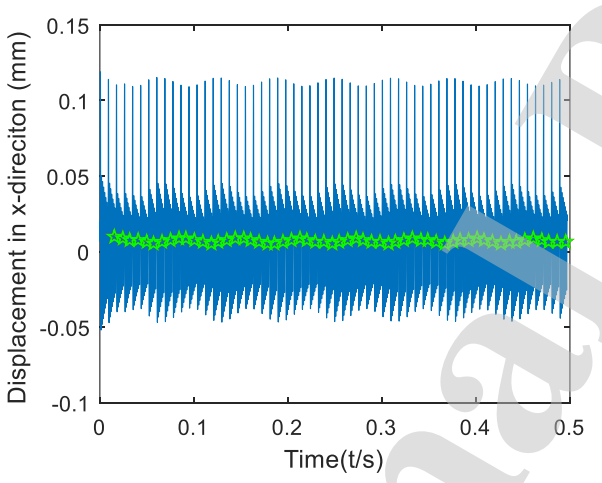

(a)

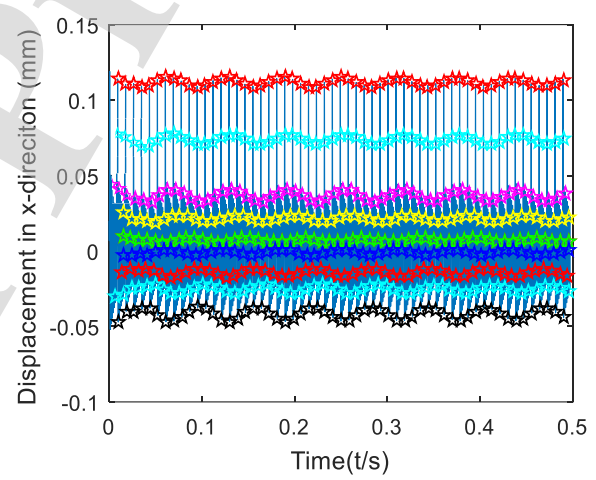

(b)

Fig. 1. (a) Once-per-revolution sampled data; and (b) MSPR - Multiple sampling per revolution data $(M=9)$.

Fig. 1(a) and (b) illustrate the once-per-revolution sampled data and MSPR data $(M=9)$, respectively. Due to the nine samples collected in one revolution, it can be found that the MSPR data includes nine sets of once-per-revolution sampled data. Compared with the once-per-revolution sampled data, the MSPR data has more samples to depict the chatter character. Moreover, in MSPR data, the fluctuations in each set of once-per-revolution sampled data are different. For instance, several sets of once-per-revolution sampled 
data, which are close to zero, have smaller fluctuations than those far from zero. This phenomenon is the physical nature of the nonlinear models [27]. Kolokolov et al. [42] reported a similar phenomenon. For the period-2 bifurcation, different sets of once-per-revolution sampled data express quite different information. For instance, one set of once-per-revolution sampled data shows the characteristic of period-2 bifurcation. However, another set of once-per-revolution sampled data shows the characteristic of the stable process. From Fig. 1(b), one can find that each set of once-per-revolution sampled data depicts partial information of the chatter character. In this situation, it is easy to find the limitation of the once-per-revolution sampled data for early chatter detection. For the same chatter signal, the different datasets sampled by the once-perrevolution sampling express different degrees of fluctuation. Thus, the dataset sampled by the once-perrevolution sampling fails to depict the chatter character adequately. Moreover, the selection of a dataset sampled by the once-per-revolution sampling affects the accuracy of the early chatter detection. In other words, in noise background, the early chatter is not detectable reliably using one set of once-per-revolution sampled data. The MSPR can overcome the shortcoming of once-per-revolution sampling.

\section{Second-order reliability method based on the MaxEnt feature}

In this section, a reliability analysis method, i.e., SORM based on the MaxEnt feature, is introduced for developing an MFRM method of real-time detection of early chatter. The SORM based on the method of the MaxEnt feature is comparable to the moment methods in structural reliability [43]. It can estimate the PDF without the difficulty of focusing on the design aspects. This difficulty spares neither the first-order reliability method nor the SORM.

\subsection{MaxEnt reliability model}

The proposed reliability model is the hardware reliability model, according to Rausand [21]. For a structure or system to be reliable, it must withstand the various loads (stresses), be they stochastic or deterministic. Let us consider, for instance, two variables: resistance (noted as $R$ ) of the structure and load (noted as $S$ ) on the structure. Here, $R$ and $S$ are stochastic variables. To satisfy the safety requirement, the resistance $R$ 
should exceed the load $S$, which can express mathematically as

$$
Z=R-S>0
$$

where $Z$ is the performance feature function.

In practice, the load may increase due to the variation of the operating conditions, or the resistance may decrease due to structural performance degradation. In this situation, the performance feature function $Z$ may be less than zero, and then failure may occur with a certain probability. For example, Fig. 2 shows the failure probability $(\mathrm{FP}, \quad p(Z<0))$ caused by structural performance degradation. By applying the SORM to the MaxEnt feature, we obtain the PDF $f(z)$, with FP defined by $p(Z<0)$, and FHF defined by $\lambda(z)$, where $z$ represents the realizations of $Z$. Referring to [21], FP and FHF express as follows:

$$
\begin{gathered}
p(Z<0)=\int_{Z<0} f_{Z}(z) d z, \\
\lambda(z)=\frac{f(z)}{1-p(z<0)}=\frac{f(z)}{R(z)},
\end{gathered}
$$

where the domain of integration $Z<0$ corresponds to failure. In the detection process modeling of early chatter, the event $Z<0$ means that the chatter feature related to the stresses of the cutting process is higher than necessary, relatively to the stresses in the chatter-free stage. The term necessary means the cutting force and dynamics does not adapt to the strength and characteristics of the material.

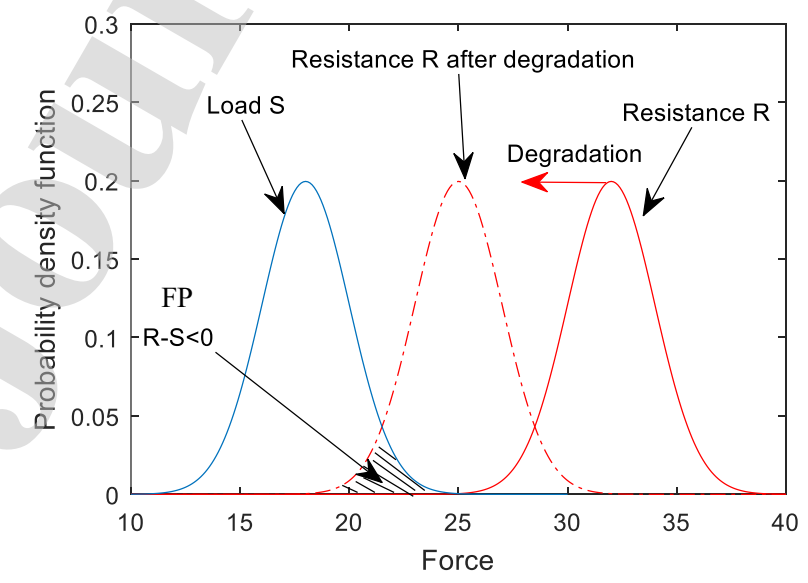

Fig. 2. FP caused by structural performance degradation. 
The early chatter can be detected using an indicator from the MaxEnt feature reliability model, starting from a load-resistance-like analysis. The chatter feature extracted from the vibration signal would represent the cutting force produced by the tool in the milling process. The vibration signal contains relevant information on the stochastic chatter process as a result of this analysis. So one can use the MaxEnt feature as an indicator of this information, a random variable modeled by $Z$, extracted from the vibration signal packets. Consider $z$ represents the realizations of the random variable $Z$ that describes the stochastic chatter process; one can then estimate the failure rate and calculate the corresponding FHF related to the chatter process. Comparing obtained FHF to a preset threshold $\mathrm{FHF}_{0}$ will allow detecting early chatter.

Let us consider the sampling domain as described in [44] for the system FP. Intuitively, a component operating in a design range close to the limit state may cross this limit to the failure region when subjected to a stochastic process such as the milling process [45].

Therefore, by increasing the number of data sampled on a milling machine, the analysis makes it possible to reach the risk domain included in the sampling domain defined by Harnitz [44] based on the SORM, which would not have been otherwise.

It is necessary to calculate the FHF as the chatter indicator, to conduct the early chatter detection based on the SORM. Thus, the following section illustrates the calculation of the FHF.

\subsection{Calculation of FHF based on the MaxEnt feature}

The signal is segmented suitably into segments or time windows for detecting the early chatter reliably and quickly. The size of the segment is an essential parameter to trade-off the reliability and the detection delay. Here, the size of the segment refers to the number of revolutions in one segment. The large size of the segment leads to a significant detection delay. However, the small size of the segment does not provide sufficient information generally. Fortunately, the MaxEnt principle is a significant breakthrough in estimating the probability density function (PDF) [46]. Under the condition of insufficient information, the estimated PDF based on the MaxEnt feature is the optimized and the most unbiased one among numerous 
candidates satisfying given conditions.

In many studies, the MaxEnt principle, with the first four moments, performs well to describe a wide range of distribution types. Of course, the estimation of the PDF is more accurate when higher-order moments are accommodated. However, the use of higher-order moments may lead to the occurrence of instability in estimating the PDF. We have checked the MaxEnt principle with the first five/six moments. They do produce the instability phenomenon. Therefore, we propose to use the MaxEnt feature with the first four moments to estimate the PDF (see Appendix for details). So, one can calculate the FHF using equation (6) and (7) based on the estimated PDF for early chatter detection.

\section{The proposed MFRM method for real-time detection of early chatter}

Generally, the chatter signal in high-speed milling process includes three parts: the periodic components due to the intermittent milling and the rotation of the cutting tool, the chatter component due to the regenerative effect, and the noise component from the measuring instrument, in-homogeneous material, and some uncontrollable influences [25]. The critical task for the early chatter detection is to find out the chatter component quickly and reliably. Up to now, most of the work for detecting the chatter focuses on filtering the periodic component and the noise component for highlighting the chatter component. However, as mentioned in Section 1, most of the filtering procedures are not suitable for early chatter detection. Also, few researchers consider the slight intolerable chatter detection, which is necessary for special precision machining. Therefore, this work proposes an MFRM method to detect two kinds of early chatter based on the SORM with MSPR. The proposed MFRM method can detect the early stage of the severe chatter, and the slightly intolerable chatter. The chatter is a kind of self-excited vibration phenomenon. Thus, the use of vibration signal is reasonable to detect early chatter. Fig. 3 illustrates the proposed MFRM method.

\section{Step 1: Set prior knowledge}

In the early chatter detection, the sampling frequency, the size of the segment, the reference feature, and the threshold $\mathrm{FHF}_{0}$ need to be set in advance. In order to obtain MSPR data conveniently, the sampling 
frequency not only satisfies the Nyquist-Shannon sampling theorem but also should be an integral multiple of spindle rotation frequency. The high sampling frequency means more information obtained in one revolution, for characterizing the chatter more adequately. However, the high sampling frequency also means a high sampling cost. Thus, in practice, the sampling frequency is determined by considering the sampling cost and accuracy in depicting the chatter character. The size of the segment is the number of revolutions in one segment and impacts the accuracy and sensitivity of chatter detection. The small size of the segment allows reducing the detection delay and obtaining high sensitivity. However, the small size of the segment does not reflect the chatter characters sufficiently and then impact the detection accuracy. Considering the two different aspects and their trade-off, we recommend that the size of the segment equals 20 to 30 revolutions.

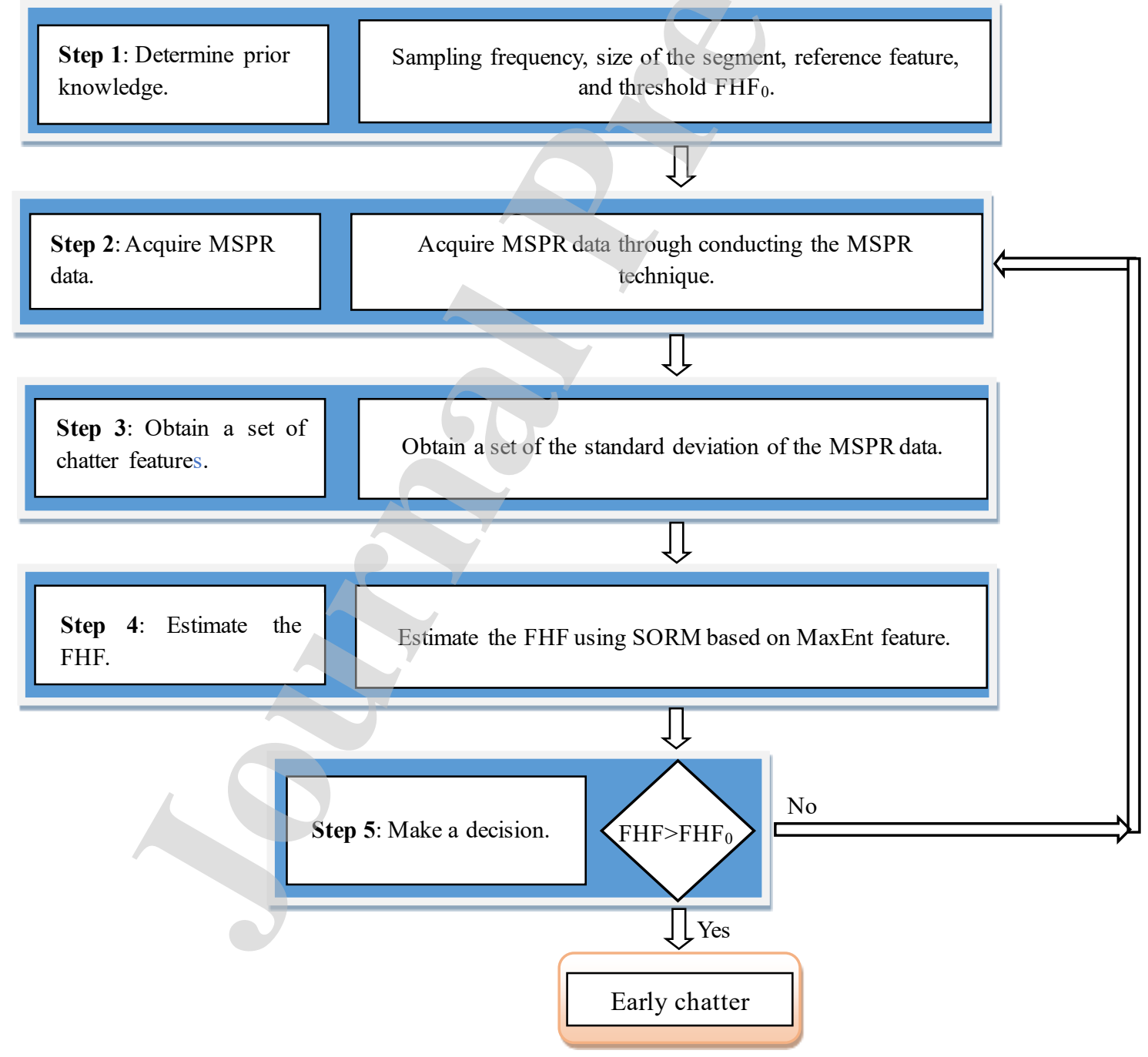

Fig. 3. Flowchart of the proposed MFRM method. 
Let us consider as reference feature, the first four moments of the standard deviation of the MSPR data at the chatter-free stage. Referring to [26][47], one can determine the reference feature. After determining the reference feature, one can also determine the relating threshold $\mathrm{FHF}_{0}$ based on reliability analysis and the $3 \delta$ principle [3]. If the estimated $\mathrm{FHF}$ is less than the $\mathrm{FHF}_{0}$, it means the milling process is in control. On the contrary, the crossing of the FHF over the preset threshold reveals the appearance of the early chatter with a probability of $99.9 \%$.

Step 2: Acquire MSPR data

Collect the vibration signal using the MSPR in real-time, and then acquire MSPR data.

Step 3: Obtain a set of chatter feature

Calculate the standard deviation of each line of MSPR data as chatter feature, and then obtain a set of chatter features.

Step 4: Estimate the FHF

Assume the standard deviation at the chatter-free stage as the resistance $R$ and the standard deviation of MSPR data in the most recent cutting process as the load $S$, and then obtain the FHF by using the SORM based on MaxEnt feature. The obtained FHF is the chatter indicator.

Step 5: Make a decision

Compare the obtained FHF and the setting threshold $\mathrm{FHF}_{0}$. If the FHF is less than or equal to $\mathrm{FHF}_{0}$, it means the cutting process is in control. The detection procedure returns to the second step and continues to monitor the milling process. Otherwise, the milling process is out of control, and the early chatter has appeared and been detected.

\section{Numerical validation and discussion}

To validate the effectiveness of the proposed MFRM method, we choose two examples relating to two kinds of early chatter detection. 


\subsection{Numerical validation I}

Referring to the components of a chatter signal, we used a reference signal based on the model in [25] with varying frequencies, and to which we added harmonic and noise components to simulate the chatter. For simplicity, we assume that the variation of the chatter component does not affect the periodic components due to the intermittent milling and the rotation of the cutting tool. The simulated chatter signal incorporating seven components is expressed as:

$$
\begin{gathered}
y=4 \sin (800 \pi t)+1.5 \sin (1600 \pi t)+4 \sin (2400 \pi t) \\
+A(t)(1+0.3 \sin (1995 \pi t)) \cos (8991 \pi t) \\
+\sin (4800 \pi t)+0.6 \sin (7200 \pi t)+w(t)) \\
A(t)=5 t .
\end{gathered}
$$

The first component is the sinusoidal wave with relatively low frequency representing the spindle rotation frequency 24,000 rpm. The second component represents the second harmonic of the spindle rotation frequency. The third component is a sinusoidal signal with three times the spindle rotation frequency, which corresponds to the tooth passing frequency and the third harmonic of the spindle rotation frequency. So, it implies that the cutting tool has three flutes. Amplitude and phase modulation components are designed as the fourth component to represent the chatter, which is a typical modulation. Here, the amplitude $A(t)$ is a time-varying function and represents the evolution of the chatter with time. The fifth and sixth components are harmonics of the spindle rotation frequency and tooth passing frequency. The seventh component represents the noise of the signal. The signal to noise ratio (SNR) is assumed as $10 \mathrm{~dB}$. The simulated signal without the chatter component represents the chatter-free signal.

In practice, the noise is inevitable and impacts the character of the dataset sampled using once-perrevolution sampling. Fig. 4(a) and (b) show the time domain signals with the noise at the chatter-free and chatter stages, respectively. The red points represent the dataset sampled once-per-revolution. It can be seen that the appearance of noise increases the difficulty of the early chatter detection.

The MSPR data is obtained by using the MSPR technique described in Section 2. According to the 
description of [16][17], the once-per-revolution sampled data at the chatter-free stage has narrow distribution. On the other hand, the distribution of the once-per-revolution sampled data at the chatter stage is wider. Indeed, the MSPR data consists of several datasets sampled once-per-revolution. They depict the chatter character in more detail than once-per-revolution sampled data. Thus, the MSPR data can improve the reliability of the early chatter detection.

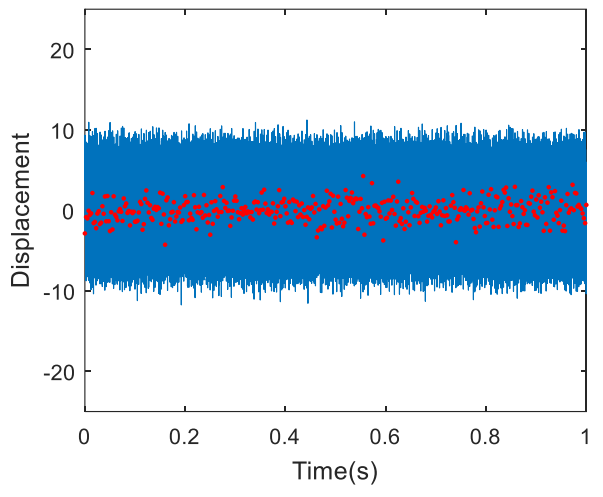

(a)

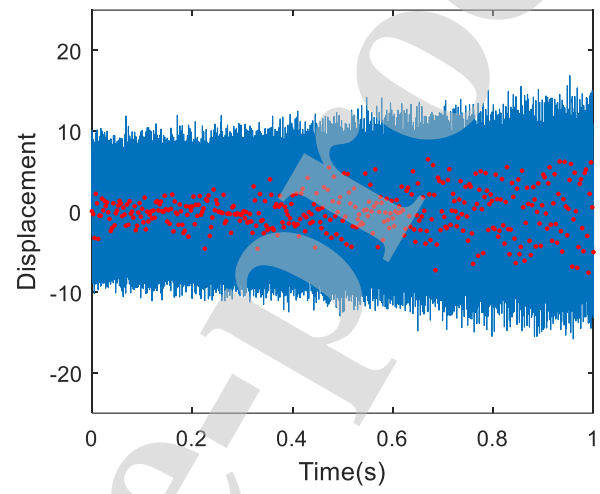

(b)

Fig. 4. The once-per-revolution sampled data of the displacement with the noise, at the stage of (a) chatterfree; (b) chatter.

The calculation of the standard deviation of the MSPR data yields a set of standard deviation. Following the SORM theory, one can assume the resistance variable $R$ corresponds to the standard deviation of the MSPR data at the chatter-free stage, and the load variable $S$ corresponds to the standard deviation of the MSPR data in the most recent cutting process. Thus, we apply the SORM for detecting the early chatter with better accuracy by estimating the FHF based on the MaxEnt feature. In the proposed MFRM method, the reference feature is preset according to several experiments or expert knowledge. Table I lists the preset reference feature, i.e., the first four moments of the standard deviation of the MSPR data using equation (11) at the chatter-free stage.

Table I. Reference feature (the first four moments of the standard deviation in the MSPR data at the chatter-free stage).

\begin{tabular}{ccccc}
\hline Moments & First & Second & Third & Fourth \\
\hline Values & 1.7008 & 0.1526 & 0.0634 & 0.1904 \\
\hline
\end{tabular}

Based on the first four moments of the standard deviation of the MSPR data at the chatter-free stage and in 
the most recent milling process, the first four moments of the performance feature function are derived according to equation (13). After that derivation of the first four moments of the performance feature function, the performance feature function can be estimated by using SORM based on the MaxEnt feature. Fig. 5(a) and (b) shows the estimated performance feature function at the chatter-free and chatter stages, respectively. Here, we only show the first eight segments. The size of each segment is 25 revolutions. For the chatter-free stage, the performance feature function in eight segments is almost the same. There is a slight difference caused by noise. When the intensity of the chatter increases, the performance feature function shifts to the left gradually. The verticals represent the zero of the performance feature function in Fig. 5(a) and (b). The left side of the vertical means $Z<0$, and the left covered area of the performance feature function is the probability $p(Z<0)$ called FP. Based on the estimated PDF and the FP, we can calculate the FHF to indicate the appearance of the chatter.

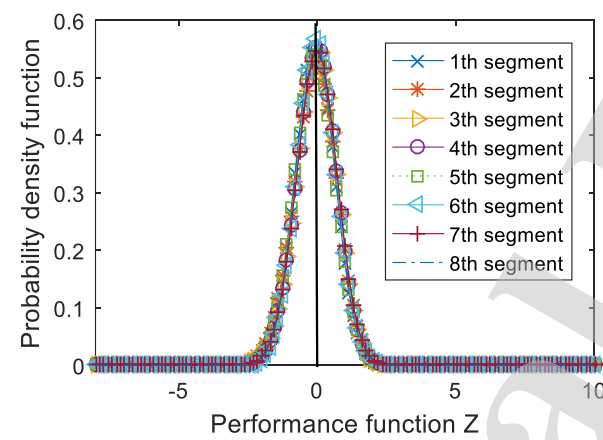

(a)

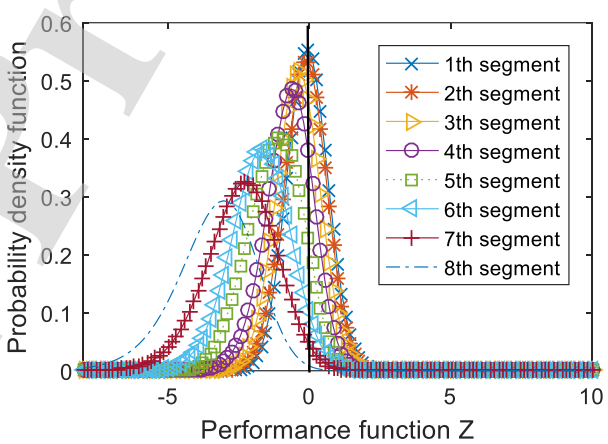

(b)

Fig. 5. Performance feature function at (a) the chatter-free stage, (b) the chatter stage.

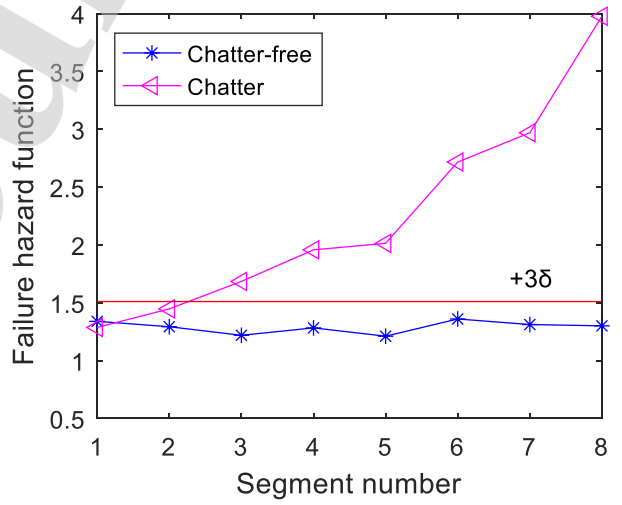

Fig.6. FHF at the chatter-free and chatter stages. 
Fig. 6. shows the FHF at the chatter-free and chatter stages. The blue curve with star symbols plots the FHF at the chatter-free stage. The FHF has a slight fluctuation caused by the noise. The pink curve with triangle symbols depicts the evolution of the FHF for the signal embodying chatter. The performance feature function gradually drifts to the left, as Fig. 5(b) shows, with the increase in FHF. The red line represents the threshold $\mathrm{FHF}_{0}$ determined based on the $3 \delta$ principle. The FHF, at the chatter-free stage, is all under the threshold, and one can claim that the milling process is in control, while for the increasing chatter, the third point crosses the red line. It means the early chatter has appeared in the third segment. The run-time of the chatter detection procedure on three segments, on a desktop computer with Intel Core i5-6600 3.3GHzCPU, is about $0.045 \mathrm{~s}$, which shows the early stage of severe chatter can be detected quickly.

\subsection{Numerical validation II}

This subsection will allow us to evaluate the effectiveness of the proposed MFRM method for detecting the early stage of a slightly intolerable chatter. For that purpose, a high-speed milling simulation model with the bearing clearance fault in [48] is chosen to generate the vibration signal, as depicted in Fig. 7. The cutting tool is equivalent to a two-degree-of-freedom (2-DOF) dynamic system in the feed ( $x-$-) and crossfeed $(y-)$ directions. The work-piece is assumed rigid. The variable stiffness represents the effect of the bearing clearance fault on the dynamic system [48][49].

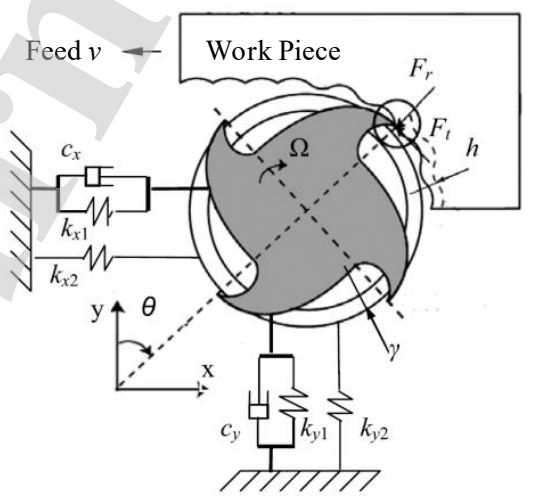

Fig. 7. A dynamic model of the milling process with bearing clearance fault [48].

The 2-DOF dynamic milling system is excited by two variable cutting forces in $x$-direction and $y$-direction. According to D'Alambert principle, its dynamic governing equations are as it follows: 


$$
\left\{\begin{array}{l}
m_{x} \ddot{x}+c_{x} \dot{x}+k_{x 1}+\delta(D-\gamma) k_{x 2}(D-\gamma) \cos (\varphi)=F_{x}(t) \\
m_{y} \ddot{x}+c_{y} \dot{x}+k_{y 1}+\delta(D-\gamma) k_{y 2}(D-\gamma) \cos (\varphi)=F_{y}(t)
\end{array},\right.
$$

where $x, \dot{x}, \ddot{x}, y, \dot{y}$ and $\ddot{y}$ are the displacement, velocity, and acceleration of the cutting tool tip in $x$-direction and $y$-direction, respectively. The $m_{x}, c_{x}, \mathrm{k}_{\mathrm{x}_{1}}+\delta(\mathrm{D}-\gamma) \mathrm{k}_{\mathrm{x}_{2}}, m_{y}, c_{y}$ and $\mathrm{k}_{\mathrm{y}_{1}}+\delta(\mathrm{D}-\gamma) \mathrm{k}_{\mathrm{y}_{2}}$ are the model mass, model damping, and model stiffness in $x$-direction and $y$-direction, respectively. Where, $D=\sqrt{x^{2}+y^{2}}, \cos (\phi)=x / D, \sin (\phi)=y / D$, and $\gamma$ is the radial clearance of the bearing, and $\delta(\cdot)$ is a switch function with $\delta(\Delta)=1$, if the $\Delta \geq 0$, and $\delta(\Delta)=0$, if the $\Delta<0$. Table II displays the model parameters of the dynamic system. $F_{x}(t)$ and $F_{y}(t)$ represent the variable cutting force at time $t$ in $x$-direction and $y$-directions and are modeled based on the regeneration effect [18][50].

Table II. Model parameters of the cutting tool in $x$-direction and $y$-direction.

\begin{tabular}{cccc}
\hline & Mass $(\mathrm{kg})$ & $\begin{array}{c}\text { Stiffness }\left(k_{1}+k_{2}\right) \\
(\mathrm{N} / \mathrm{s})\end{array}$ & $\begin{array}{c}\text { Damping ratio } \\
(\%)\end{array}$ \\
\hline$x$ & $2.75 \times 10^{-2}$ & $8.79 \times 10^{5}$ & 1.39 \\
$y$ & $2.96 \times 10^{-2}$ & $9.71 \times 10^{5}$ & 1.38 \\
\hline
\end{tabular}

The carbide square end-cutting tool with a diameter of $12 \mathrm{~mm}$, single flute, and helix angle $30^{\circ}$ is chosen to cut the work-piece. The set machining parameters are shown in Table III, respectively. The material of the work-piece is 6061-T6 aluminum.

Table III. Machining parameters.

\begin{tabular}{cccc}
\hline $\begin{array}{c}\text { Spindle speed } \\
(\mathrm{rpm})\end{array}$ & $\begin{array}{c}\text { Axial depth } \\
(\mathrm{mm})\end{array}$ & $\begin{array}{c}\text { Radial depth } \\
(\mathrm{mm})\end{array}$ & $\begin{array}{c}\text { Feed per tooth } \\
(\mathrm{mm} / \text { tooth })\end{array}$ \\
\hline 7000 & 3 & 0.5 & 0.1 \\
\hline
\end{tabular}

The dynamic response of the cutting tool is simulated in the time domain [51]. Fig. 8(a) and (b) show the displacement of the cutting tool in the $x$-direction and the Poincaré map without the bearing clearance fault. The red stars represent the once-per-revolution sampled data. It is clear from the two figures that the onceper-revolution sampled data is constant, which means the cutting process is stable.

During the milling process, the clearance fault may appear in the bearing of the spindle. Under the condition of the bearing clearance fault $\gamma=4 \mu m$, the cutting process is simulated and plotted in Fig. 9(a) and (b). 
One can notice, the once-per-revolution sampled data is not constant anymore and has a slight fluctuation.

A group of points appears on the Poincaré map. These phenomena reveal the appearance of the slight chatter.

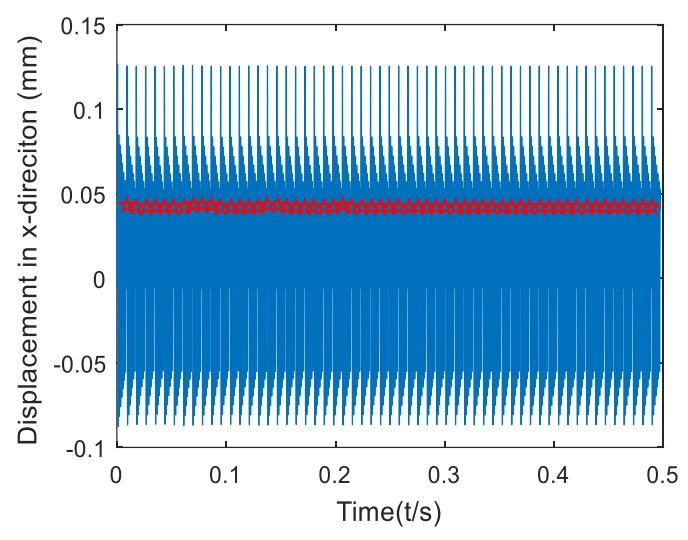

(a)

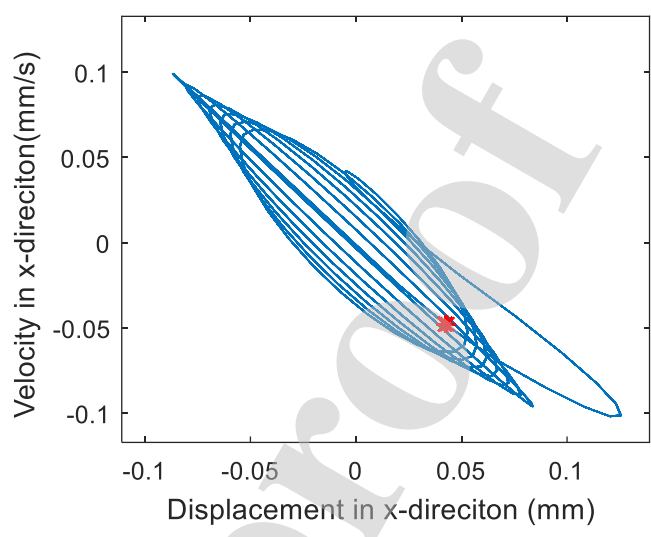

(b)

Fig. 8 Displacement response of the cutting tool at the chatter-free stage, (a): Displacement of the cutting tool in the $x$-direction, (b): Poincaré map of the cutting tool in the $x$-direction.

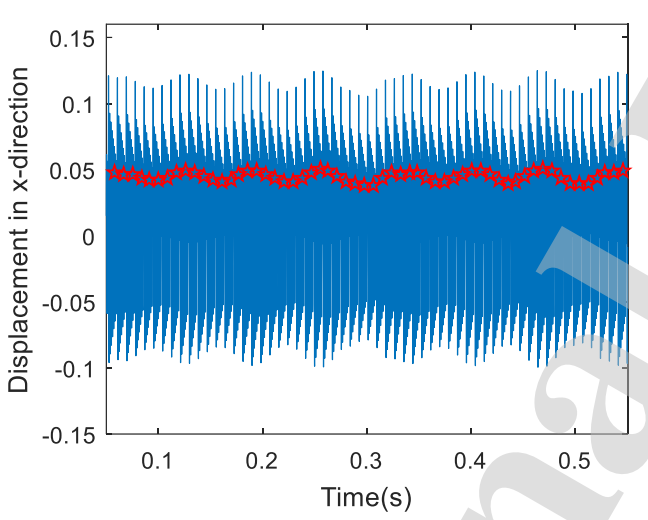

(a)

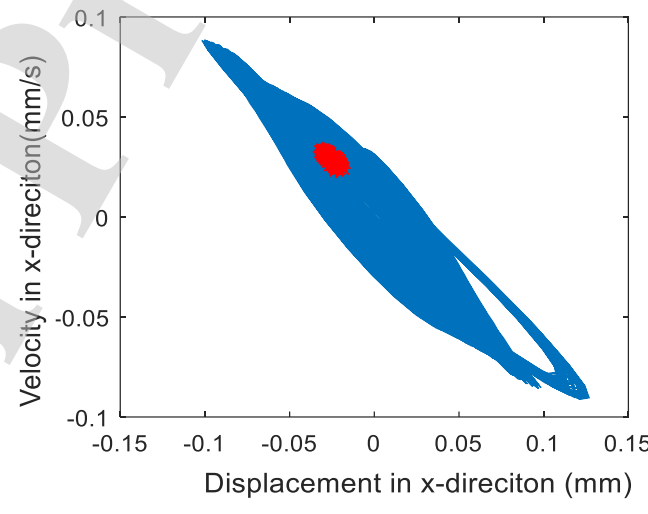

(b)

Fig. 9 Displacement response of the cutting tool at the slight chatter stage, (a): displacement of the cutting tool in the $x$-direction, (b): Poincaré map of the cutting tool in the $x$-direction.

In practice, noise emerges in all machining and measuring process and affects the once-per-revolution data. To simulate the noisy background, we add white noise to the generated cutting signal with the set SNR of $10 \mathrm{~dB}$. Fig. 10(a) and (b) show the effect of the noise on the displacement of the cutting tool at the chatterfree and slight chatter stages, respectively. The occurrence of noise changes the distribution of the onceper-revolution sampled data. The difference between the two distributions is not clear anymore. 
After collected the MSPR data, we calculated the relating standard deviation and got the chatter feature.

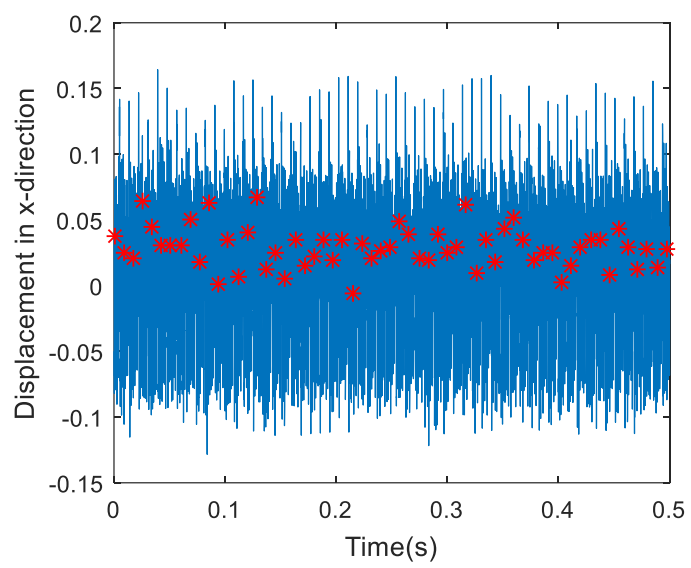

(a)

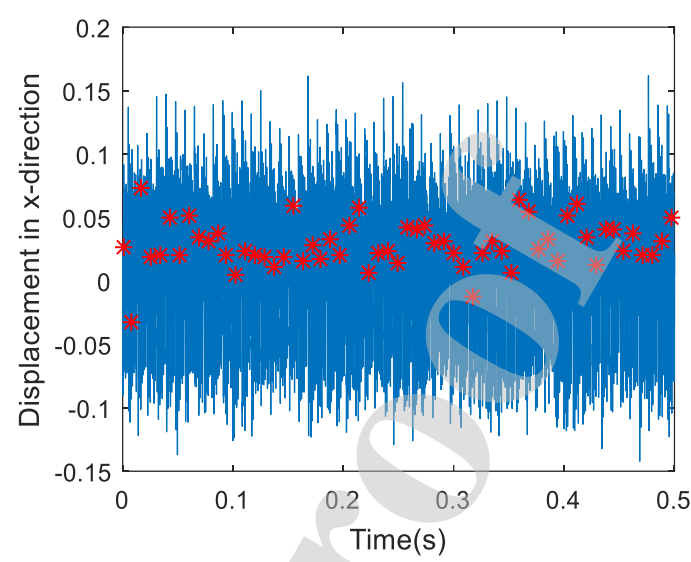

(b)

Fig. 10. Effect of the noise on the displacement of the cutting tool at the chatter-free and slight chatter stages, (a) Chatter-free, (b) slight chatter.

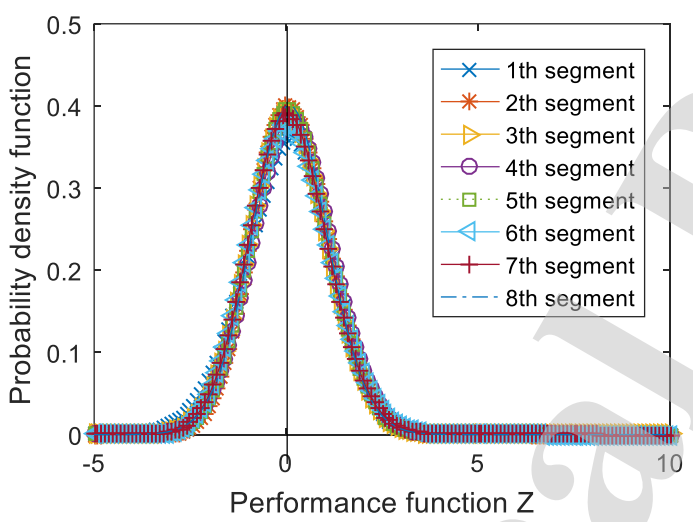

(a)

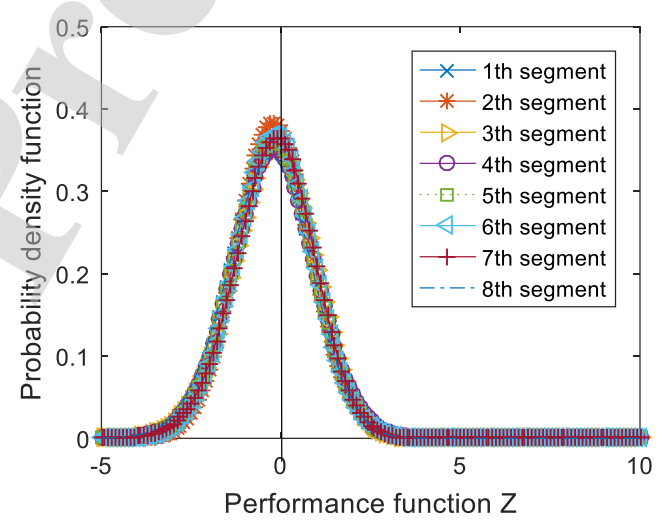

(b)

Fig. 11. Performance feature function at the chatter-free and slight chatter stages, (a) at the chatter-free stage, (b) at the slight chatter stage.

The first four moments of the standard deviation of the MSPR data in the most recent cutting process are estimated according to equation (11). Based on these estimated first four moments and the preset reference feature, one can derive the first four moments of the performance feature function according to (13). We used the SORM based on the MaxEnt feature to estimate the performance feature function and to calculate the FP. Fig. 11(a) and (b) show the estimated performance feature function at the chatter-free and slight chatter stages, respectively. We display hereafter, the eight segments for each situation, with segments sizes 
chosen equal to 25 . The verticals in Fig 11 (a) and (b) pass through $Z=0$. The probability $p(Z<0)$, i.e., the covered area of the performance feature function in the left side of the vertical, is FP. According to the estimated PDF and the FP, we can calculate the FHF to detect early chatter.

Fig. 12 shows the FHF at the chatter-free and slight chatter stages. The blue curve with star symbols shows the FHF with fluctuations at the chatter-free stage. The fluctuation is caused by noise. The red line represents the threshold $\mathrm{FHF}_{0}$ defined based on the $3 \delta$ principle. For the chatter-free process, the FHF is below the threshold $\mathrm{FHF}_{0}$, which means the cutting process is in control. The pink curve with the triangle symbols depicts the FHF at the slight chatter stage. Some values of the FHF have crossed the threshold $\mathrm{FHF}_{0}$. Thus, one can say that the slight chatter has appeared, and claim that the proposed MFRM method can detect the slight chatter effectively.

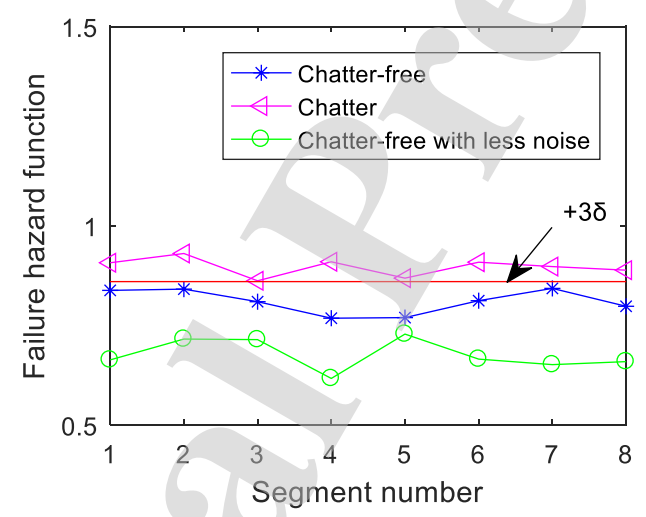

Fig. 12. FHF at the chatter-free and chatter stages.

Besides, we compared the proposed indicator of FHF with the Kullback-Leibler (KL) divergence. The KL divergence is a tool to compare two probability distributions. By the definition of the KL divergence, the difference will reach zero if the two probability distributions are the same. For two different probability distributions, the KL divergence is not equal to zero. Furthermore, the KL divergence increases with the difference between the two probability distributions. The KLdivergence has been applied for fault detection in dynamic systems [52]. For the early chatter detection, we can measure the difference between the estimated and the preset PDF. However, the KL divergence may not indicate the appearance of the chatter reliably since the KL divergence cannot distinguish the relative location of the two probability distributions. 
The KL divergences at the chatter-free and early chatter stages are shown in Fig. 13. There are two cases at the chatter-free stage. The first one is that the adding SNR is $10 \mathrm{~dB}$. The second one has less noise, and its SNR is $10.5 \mathrm{~dB}$. According to Fig. 13, one can find that KL divergence fails to detect chatter. The decrease in the embedded noise leads to a false alarm. During the degradation of a new cutting tool, the produced noise decreases first and then increases. The KL divergence cannot meet this situation in detecting chatter. The proposed FHF can overcome the shortage of the KL divergence, as depicted in Fig.12. The decrease in the embedded noise does not lead to a false alarm.

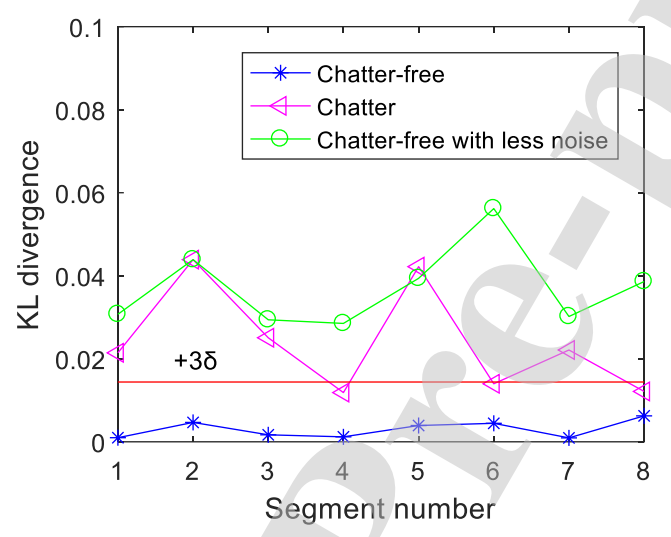

Fig. 13. KL divergence at the chatter-free and chatter stages.

The experimental results have demonstrated the efficiency of the proposed MFRM method for real-time detection of early chatter. This method can detect early chatter reliably by employing SORM and MSPR. In the proposed MFRM method, the process of estimating PDF based on the MaxEnt feature takes most of the running time. Therefore, we introduced a numerical method for estimating the PDF first. Then, we analyzed the time complexity of the MaxEnt principle with the first four moments.

The estimated PDF based on the MaxEnt feature with the first four moments is represented by equation (21) with the Lagrange multipliers, which can be computed by solving the nonlinear equations (18). However, it is impossible to obtain an analytical solution for a general case. Therefore, some iterative algorithms are proposed to determine the approximate solution, such as Newton's method, gradient descent, and improved iterative scaling. In this work, we employed Newton's method to solve the nonlinear equations (18). Generally, the procedure consists of three steps as follows: expend the nonlinear equations (18) in Taylor's 
series around trial values of $\lambda$, drop the quadratic and higher-order terms, and solve the remaining linear equations iteratively [53]. The iteration process continues until a preset error is reached. According to the first four moments used in this work, there are 13 integrals to be calculated for determining Lagrange multipliers in each iteration process. The univariate Simpson's method is used to calculate these integrals.

It is necessary to consider the time complexity of the MaxEnt principle for real-time chatter detection. Since the iteration needs to be produced by using Newton's method for satisfying the preset error and integrals need to be calculated by using univariate Simpson's method, we relied on the time complexity study of these two methods for analyzing the complexity of the MaxEnt.

Using the common notation $O$ in computer science for expressing the time complexity of an algorithm, the time complexity for the univariate Simpson's method is $O(q)$, where $q$ is the number of subdivisions between up and down bounds of integration. Besides, the time complexity of determining Lagrange multipliers using the Newton's method with specified precision depends on the given initial approximation and the application.

Lastly, the time complexity of the MaxEnt principle with the first four moments is acceptable for real-time chatter detection. Although the computational cost of the MaxEnt principle is high, it is used for complex models with several variables, such as in language processing, for instance [54]. For models involving a few variables, peculiarly one variable, the computational cost of the MaxEnt principle is low. The proposed method with the MaxEnt feature-based reliability model considers only one variable. Then, the computational process has low complexity. So the time complexity of the MaxEnt principle with the first four moments is acceptable for real-time chatter detection.

To highlight the computational cost of the proposed MFRM method, we have assessed the chatter detection procedure on a desktop computer with Intel Core i5-6600 3.3GHz CPU. The computational cost with 25 size segments is about $0.015 \mathrm{~s}$. It is much less than the machine run-time $0.0625 \mathrm{~s}$ (the first experiment with the spindle revolution speed of $24,000 \mathrm{rpm}$ ) and $0.2143 \mathrm{~s}$ (the second experiment with the spindle revolution 
speed of 7,000 rpm) in a segment.

For the different machining materials and measuring instruments, the noise embedded in the signal may be different. Under the given reference feature, less noise may lead to the missing chatter alarm or increases the detection delay because the less noise decreases the standard deviation of the MSPR data. Similarly, more significant noise may cause the fault chatter alarm. Aimed at these problems, one can choose a variable reference feature or adaptive threshold $\mathrm{FHF}_{0}$ to adapt to the variation of the noise.

The chatter relates to an unstable phenomenon (bifurcation or chaos processes). The unstable phenomenon exists in many nonlinear dynamic systems, such as rotor-bearing systems and pulses energy conversion systems. In the rotor-bearing system, the degradation of the component leads to the appearance of the unstable phenomenon [55]. The early degradation detection of component is an essential task for the fault diagnosis and prognosis [56]. The proposed method with the MaxEnt feature-based reliability model is suitable to monitor the incipient degradation of a component by detecting the occurrence of instability. According to the literature [35][36], the boundary between the stable and unstable area is a region instead of a line. In this region, the bifurcation phenomenon may have intermittent property, i.e., alternation of stable and unstable phenomena appears. For this case, the proposed MFRM method is also suitable, due to its property of rapidity and reliability.

\section{Conclusions}

In this work, we proposed an MFRM method to detect two kinds of early chatter, i.e., the early stage of the severe chatter and slightly intolerable chatter. The proposed MFRM method for real-time detection of early chatter is based on the MSPR and SORM. The early chatter detection procedure is implemented by using the reliability approach. The MSPR enhances the reliability of the once-per-revolution sampling strategy by exploring the data resource. The SORM allows deriving the indicator FHF for a reliable early chatter detection. With the MaxEnt feature, SORM avoids the difficulty of focusing on the design point.

Moreover, the proposed MFRM method is independent of machining parameters and has a less 
computational cost. Besides detecting early chatter, the proposed MFRM method can be used to monitor the degradation in rotor-bearing systems and the instability in pulse energy conversion systems.

Although the proposed MFRM method detects two kinds of early chatter effectively, there is a limitation. In the milling process, the transient behavior caused by the cutting tool entry/exit affects the chatter feature of MSPR data. It then reduces the detection accuracy of the proposed MFRM method. In the future, our work will address this limitation by working out other approaches and extend the application area in early fault detection.

\section{Acknowledgments}

This work was supported by the Nature Science Foundation of Huai'an city of China (Grant No.HAB201834).

\section{References}

[1] Quintana G, Ciurana J. Chatter in machining processes: A review. Int J Mach Tools Manuf 2011;51:363-76. https://doi.org/10.1016/j.ijmachtools.2011.01.001.

[2] Zhu L, Liu C. Recent progress of chatter prediction, detection and suppression in milling. Mech Syst Signal Process 2020;143:106840. https://doi.org/10.1016/j.ymssp.2020.106840.

[3] Cao H, Zhou K, Chen X, Zhang X. Early chatter detection in end milling based on multi-feature fusion and $3 \sigma$ criterion. Int J Adv Manuf Technol 2017;92:4387-97. https://doi.org/10.1007/s00170017-0476-x.

[4] Jia G, Wu B, Hu Y, Xie F, Liu A. A synthetic criterion for early recognition of cutting chatter. Sci China Technol Sci 2013;56:2870-6. https://doi.org/10.1007/s11431-013-5360-9.

[5] Hynynen KM, Ratava J, Lindh T, Rikkonen M, Ryynänen V, Lohtander M, et al. Chatter detection in turning processes using coherence of acceleration and audio signals. J Manuf Sci Eng 2014;136:044503. https://doi.org/10.1115/1.4026948.

[6] Yao Z, Mei D, Chen Z. On-line chatter detection and identification based on wavelet and support 
vector machine. J Mater Process Technol 2010;210:713-9. https://doi.org/10.1016/j.jmatprotec.2009.11.007.

[7] Yang K, Wang G, Dong Y, Zhang Q, Sang L. Early chatter identification based on an optimized variational mode decomposition. Mech Syst Signal Process 2019;115:238-54. https://doi.org/10.1016/j.ymssp.2018.05.052.

[8] Rusinek R, Pawel L, Krzysztof K, Kruszynski B, Warminski J. Chatteridentification methods on the basis of time series measured during titanium superalloy milling. Int J Mech Sci 2015;99:196-207. https://d oi.org/10.1016/j.ijmecsci.2015.05.013.

[9] Aslan D, Altintas Y. On-line chatter detection in milling using drive motor current commands extracted from CNC. Int J Mach Tools Manuf 2018;132:64-80. https://d oi.org/10.1016/j.ijmachtools.2018.04.007.

[10] Ma L, Melkote SN, Castle JB. A model-based computationally efficient method for on-line detection of chatter in milling. J Manuf Sci Eng 2013;135:031007. https://doi.org/10.1115/1.4023716.

[11] Liu C, Zhu L, Ni C. Chatter detection in milling process based on VMD and energy entropy. Mech Syst Signal Process 2018;105:169-82. https://doi.org/10.1016/j.ymssp.2017.11.046.

[12] van Dijk NJM, Doppenberg EJJ, Faassen RPH, van de Wouw N, Oosterling JAJ, Nijmeijer H. Automatic in-process chatter avoidance in the high-speed milling process. J Dyn Syst Meas Control 2010;132:031006. https://doi.org/10.1115/1.4000821.

[13] Messaoud A, Weihs C. Monitoring a deep hole drilling process by nonlinear time series modeling. J Sound Vib 2009;321:620-30. https://doi.org/10.1016/j.jsv.2008.10.028.

[14] Kakinuma Y, Sudo Y, Aoyama T. Detection of chatter vibration in end milling applying disturbance observer. CIRP Ann - Manuf Technol 2011;60:109-12. https://doi.org/10.1016/j.cirp.2011.03.080.

[15] Caliskan H, Kilic ZM, Altintas Y. On-line energy-based milling chatter detection. J Manuf Sci Eng Trans ASME 2018;140:1-12. https://doi.org/10.1115/1.4040617.

[16] Schmitz TL, Medicus K, DuttererB. Exploring once-per-revolution audio signal variance as a chatter indicator. Mach Sci Technol 2002;6:215-33. https://doi.org/10.1081/MST-120005957. 
[17] Schmitz TL. Chatter recognition by a statistical evaluation of the synchronously sampled audio signal. J Sound Vib 2003;262:721-30. https://doi.org/10.1016/S0022-460X(03)00119-6.

[18] Honeycutt A, Schmitz TL. A new metric for automated stability identification in time domain milling simulation. J Manuf Sci Eng 2016;138:1-7. https://doi.org/10.1115/1.4032586.

[19] Du R, Elbestawi MA, Ullagaddi BC. Chatter detection in milling based on the probability distribution of cutting force signal. Mech Syst Signal Process 1992;6:345-62. https://doi.org/10.1016/0888-3270(92)90036-I.

[20] Deng C, Miao J, Ma Y, Wei B, Feng Y. Reliability analysis of chatter stability for milling process system with uncertainties based on neural network and fourth moment method. Int J Prod Res 2019;58:2732-50. https://doi.org/10.1080/00207543.2019.1636327.

[21] Rausand M, Hoyland A. System reliability theory: models, statistical methods, and applications. 2nd Ed. John Wiley \& Sons, Inc.; 2004.

[22] Govekarl E, GradiSek J, Kalveram M, Insperger T, Weinert K, Stepan G, et al. On stability and dynamics of milling at small radial immersion. CIRP Ann 2005;54:357-62.

[23] Davies M, Pratt JR, B. S D, T. J B. The stability of low radial immersion milling. CIRP Ann 2000;49:37-40.

[24] Insperger T, Mann BP, Surmann T, Stépán G. On the chatter frequencies of milling processes with runout. Int J Mach Tools Manuf 2008;48:1081-9. https://doi.org/10.1016/j.ijmachtools.2008.02.002.

[25] Cao H, Lei Y, He Z. Chatter identification in end milling process using wavelet packets and HilbertHuang transform. Int J Mach Tools Manuf 2013;69:11-9. https://doi.org/10.1016/j.ijmachtools.2013.02.007.

[26] Zhao Y, Adjallah KH, Sava A, Wang Z. Early chatter detection using MaxEnt and SPRT. 2019 6th Int. Conf. Control. Decis. Inf. Technol., Paris, France: 2019, p. 1550-5. https://doi.org/10.1109/CoDIT.2019.8820670.

[27] Kolokolov Y, Monovskaya A. Fractal approach to forming of modified bifurcation diagrams in practical applications. Int J Comput 2013;12:133-41. 
[28] Liu Y, Meng L lin, Liu K, Zhang Y min. Chatter reliability of milling system based on first-order second-moment method. Int J Adv Manuf Technol 2016;87:801-9. https://doi.org/10.1007/s00170016-8523-6.

[29] Kang HY, Kwak BM. Application of maximum entropy principle for reliability-based design optimization. Struct Multidiscip Optim 2009;38:331-46. https://doi.org/10.1007/s00158-008-02993.

[30] Li H, Wen D, Lu Z, Wang Y, Deng F. Identifying the probability distribution of fatigue life using the maximum entropy principle. Entropy 2016;18. https://doi.org/10.3390/e18040111.

[31] Li C, Wang S, Wang W. Reliability analysis of free jet scour below dams. Entropy 2012;14:257888. https://doi.org/10.3390/e14122578.

[32] Li C, Wang W, Wang S. Maximum-entropy method for evaluating the slope stability of earth dams. Entropy 2012;14:1864-76. https://doi.org/10.3390/e14101864.

[33] Rajan A, Kuang YC, Ooi MPL, Demidenko S, Carstens H. Moment-constrained maximum entropy method for expanded uncertainty evaluation. IEEE Access 2018;6:4072-82. https://doi.org/10.1109/ACCESS.2017.2787736.

[34] Luigi P, Inverardi N. Maximum Entropy Density Estimation from Fractional Moments. Commun Stat Theory Methods 2003;32:327-45. https://doi.org/10.1081/STA-120018189.

[35] Zhang X, Pandey MD. Structural reliability analysis based on the concepts of entropy , fractional moment and dimensional reduction method. Struct Saf 2013;43:28-40. https://doi.org/10.1016/j.strusafe.2013.03.001.

[36] Zhang X, Min Y, Ghee C. Maximum entropy distribution with fractional moments for reliability analysis. Struct Saf 2020;83:101904. https://doi.org/10.1016/j.strusafe.2019.101904.

[37] Cheng C, Wang Z, Hung W, Bukkapatnam STS, Komanduri R. Ultra-precision machining process dynamics and surface quality monitoring. Procedia Manuf 2015;1:607-18. https://d oi.org/10.1016/j.promfg.2015.09.044.

[38] Insperger T, Stépán G, Bayly P V., Mann BP. Multiple chatter frequencies in milling processes. J 
Sound Vib 2003;262:333-45. https://doi.org/10.1016/S0022-460X(02)01131-8.

[39] Moradi H, Vossoughi G, Movahhedy MR. Bifurcation analysis of nonlinear milling process with tool wear and process damping: Sub-harmonic resonance under regenerative chatter. Int J Mech Sci 2014;85:1-19. https://doi.org/10.1016/j.jjmecsci.2014.04.011.

[40] Honeycutt A, Schmitz TL. Milling bifurcations: A review of literature and experiment. J Manuf Sci Eng Trans ASME 2018;140:1-19. https://doi.org/10.1115/1.4041325.

[41] Lu S, Yan R, Liu Y, Wang Q. Tacholess speed estimation in order tracking: A review with application to rotating machine fault diagnosis. IEEE Trans Instrum Meas 2019;68:1-18. https://d oi.org/10.1109/TIM.2019.2902806.

[42] Kolokolov Y, Monovskaya A. From modifications of experimental bifurcation diagrams to operating process stability margin. Int $\mathbf{J}$ Bifurc Chaos 2013;23:1-20. https://doi.org/10.1142/S0218127413300243.

[43] Zhao Y, Ono T. Moment methods for structural reliability considering sample size. Struct Saf 2005;23:2369-75.

[44] Harbitz A. An efficient sampling method for probability of failure calculation. Struct Saf 1986;3:109-15. https://doi.org/10.1016/0167-4730(86)90012-3.

[45] Huang X, Zhang Y, Lv C. Probabilistic analysis of dynamic stability for milling process. Nonlinear Dyn 2016;86:2105-14. https://d oi.org/10.1007/s11071-016-3019-3.

[46] Favretti M. Remarks on the maximum entropy principle with application to the Maximum Entropy Theory of Ecology. Entropy 2018;20. https://doi.org/10.3390/e20010011.

[47] Djurdjanovic D, Lee J, Ni J. Watchdog Agent-an infotronics-based prognostics approach for product performance degradation assessment and prediction. Adv Eng Informatics 2003;17:109-25. https://doi.org/10.1016/j.aei.2004.07.005.

[48] Peng ZK, Jackson MR, Guo LZ, Parkin RM, Meng G. Effects of bearing clearance on the chatter stability of milling process. Nonlinear Anal Real World Appl 2010;11:3577-89. https://d oi.org/10.1016/j.nonrwa.2010.01.005. 
[49] Wiercigroch M. Modelling of dynamical systems with motion dependent discontinuities. Chaos, Solitons and Fractals 2000;11:2429-42. https://doi.org/10.1016/S0960-0779(00)00032-1.

[50] Smith S, Tlusty J. An overview of modeling and simulation of the milling process. J Eng Ind 1991;113:169. https://doi.org/10.1115/1.2899674.

[51] Schmitz TL, Smith KS. Machining Dynamics: Frequency response to improved productivity. New York: Springer; 2009.

[52] Lei Xie; Jiusun Zeng; Uwe Kruger; Xun Wang; Jaap Geluk. Fault detection in dynamic systems using the Kullback-Leibler divergence. Control Eng Pract 2015;43:39-48. https://d oi.org/10.1016/j.conengprac.2015.05.010.

[53] Zellner A, A.Highfield R. Calculation of maximum entropy distributions and approximation of marginalposterior distributions. J Econom 1988;37:195-209.

[54] Khudanpur S, Wu J. Maximum entropy techniques for exploiting syntactic, semantic and collocational dependencies in language modeling. Comput Speech Lang 2000;14:355-72. https://d oi.org/10.1006/csla.2000.0149.

[55] Wang CC. Bifurcation analysis of an aerodynamic journal bearing system considering the effect of stationary herringbone grooves. Chaos, Solitons and Fractals 2007;33:1532-45. https://doi.org/10.1016/j.chaos.2006.03.011.

[56] Zhou F, Park JH, Wen C, Hu P. Average accumulative based time variant model for early diagnosis and prognosis of slowly varying faults. Sensors 2018;18:1-27. https://doi.org/10.3390/s18061804.

[57] Kolokolov Y, Monovskaya A. Fractal approach, bifurcation poker and SUC--logic for nonlinear dynamics forecasting. Int J Bifurc Chaos 2013;23:1-18. https://doi.org/10.1142/S0218127413502015.

[58] Kolokolov Y, Monovskaya A. Estimating the uncertainty of the behavior of a PWM power converter by analyzing a set of experimental bifurcation diagrams. Int J Bifurc Chaos 2013;23:1-14. https://doi.org/10.1142/S0218127413500636.

[59] Li Y, Zhao G. Reliability analysis of structures based on maximum entropy theory. J Dalian Univ 
Technol 1992;32:455-9. 
Appendix

We introduce the widely used MaxEnt principle with the first four moments for the estimation of the PDF. The estimated PDF is used to calculate the FHF for indicating the appearance of the chatter. The procedure of the estimation of the PDF includes three parts: calculation of moments of sample variables, estimation of the first four moments of the performance feature function, and estimation of the performance feature function.

1 Calculation of moments of sample variables

In order to estimate the performance feature function using the MaxEnt feature, it is necessary to calculate the moments of chatter features at the chatter-free stage and in the most recent cutting process. Based on the given $n$ samples of a chatter feature $x_{i}$, its mean, second, third, and fourth central moment $\left(\mu_{x_{i}, 1}, \mu_{x_{i}, 2}\right.$, $\left.\mu_{x_{i}, 3}, \mu_{x_{i}, 4}\right)$ can be estimated as follows:

$$
\left\{\begin{array}{l}
\hat{\mu}_{x_{i}, 1}=\bar{x}_{i}=\frac{1}{n} \sum_{j=1}^{n} x_{i, j} \\
\hat{\mu}_{x_{i}, 2}=\frac{1}{n} \sum_{j=1}^{n}\left(x_{i, j}-\bar{x}_{i}\right)^{2} \\
\hat{\mu}_{x_{i}, 3}=\frac{1}{n} \sum_{j=1}^{n}\left(x_{i, j}-\bar{x}_{i}\right)^{3} \\
\hat{\mu}_{x_{i}, 4}=\frac{1}{n} \sum_{j=1}^{n}\left(x_{i, j}-\bar{x}_{i}\right)^{4}
\end{array}\right.
$$

where $\hat{\mu}_{x_{i}, 1}, \hat{\mu}_{x_{i}, 2}, \hat{\mu}_{x_{i}, 3}, \hat{\mu}_{x_{i}, 4}$ are the estimated value of the $\mu_{x_{i}, 1}, \mu_{x_{i}, 2}, \mu_{x_{i}, 3}, \mu_{x_{i}, 4}$, respectively. $x_{i, j}$ is a sample, $i$ represents $i$ th the chatter feature, $j$ represents the $j$ th sample of the chatter feature $x_{i}$. For the early chatter detection, the first four moments of the chatter feature at the chatter-free stage are estimated as $\hat{\mu}_{x_{1}, 1}, \hat{\mu}_{x_{1}, 2}, \hat{\mu}_{x_{1}, 3}, \hat{\mu}_{x_{1}, 4}$. For the chatter feature in the most recent cutting process, the first four moments are estimated as $\hat{\mu}_{x_{2}, 1}, \hat{\mu}_{x_{2}, 2}, \hat{\mu}_{x_{2}, 3}, \hat{\mu}_{x_{2}, 4}$. Before the early chatter detection, the first four moments of chatter feature at chatter-free stage need to be set according to many experiments or experts' knowledge. 
2 Estimation of the first four moments of the performance feature function

According to the estimated first four moments of the chatter features, the first four moments of the performance feature function $Z$ can be estimated based on the Taylor series method. Let us express the performance feature function as $Z=g(x)=g\left(x_{1}, x_{2}, \cdots, x_{I}\right)$, where $I$ denotes the number of chatter features. Taylor series expansion of the performance feature function at the mean value expresses as [32]:

$$
Z \approx g(\mu)+\sum_{i=1}^{I} g\left(x_{i}-\mu_{x_{i}}\right)+\frac{1}{2} \sum_{i=1}^{I} \sum_{m=1}^{I} g_{i m}\left(x_{i}-\mu_{x_{i}}\right)\left(x_{m}-\mu_{x_{m}}\right),
$$

where $\mu=\left(\mu_{x_{1}}, \mu_{x_{2}}, \cdots, \mu_{x_{I}}\right), g_{i}=\frac{\partial g(\mu)}{\partial x_{i}}, g_{i m}=\frac{\partial^{2} g(\mu)}{\partial x_{i} \partial x_{m}}$

One obtains the first four moments of performance feature function $Z$ according to the following equations [59]:

$$
\left\{\begin{array}{l}
\mu_{\mathrm{Z} 1}=g(\mu)+\frac{1}{2} \sum_{i=1}^{I} g_{i i} \mu_{x_{i} 2} \\
\mu_{Z 2}=\sum_{i=1}^{I} g_{i}^{2} \mu_{x_{i} 2}+\sum_{i=1}^{I} g_{i} g_{i i} \mu_{x_{i} 3}+\frac{1}{4} \sum_{i}^{I} g_{i i}^{2}\left(\mu_{x_{i} 4}-\mu_{x_{i} 2}^{2}\right)+\frac{1}{2} \sum_{i=1}^{I} \sum_{m=1}^{M} g_{i i}^{2} \mu_{x_{i} 2} \mu_{x_{m} 2} \\
\mu_{Z 3}=\sum_{i=1}^{I} g_{i}^{3} \mu_{x_{i} 3}+\frac{3}{2} \sum_{i=1}^{I} g_{i}^{2} g_{i i}\left(\mu_{x_{i} 4}-\mu_{x_{i} 2}^{2}\right)+3 \sum_{i=1}^{I} \sum_{m=1}^{M} g_{i} g_{m} g_{i i} \mu_{x_{i} 2} \mu_{x_{m} 2} \\
\mu_{Z 4}=\sum_{i=1}^{I} g_{i}^{4} \mu_{x_{i} 4}+3 \sum_{i=1}^{I} \sum_{m=1}^{M} g_{i}^{2} g_{m}^{2} \mu_{x_{i} 2} \mu_{x_{m} 2}
\end{array} .\right.
$$

3 Estimation of the performance feature function

For a continuous stochastic variable $Z$, we assume its PDF as $f(z)$, where $z$ represents the realizations of $Z$. The first moment $\mu_{z, 1}$ is calculated as follow:

$$
\mu_{z, 1}=\int_{\Omega} z f(z) d z
$$

where $\Omega=\{-\infty,+\infty\}$ is the integral domain of the stochastic variable $Z$. The second, third and fourth central moment of the $Z$ is calculated by 


$$
\mu_{Z, j}=\int_{\Omega}\left(z-\mu_{Z, 1}\right)^{j} f(z) d z \quad(j=2,3,4)
$$

The entropy expresses as

$$
H(f(z))=\int_{\Omega} f(z) \ln f(z) d z
$$

Following the MaxEnt feature, the objective is to find the optimal $f(z)$, which maximizes the entropy $H(f(z))$ under some constraints. The MaxEnt principle estimating the optimal PDF $f(z)$ expresses as follows:

$$
\begin{gathered}
\max \quad H(f(z))=\int_{\Omega} f(z) \ln f(z) d z . \\
\text { s.t. } \quad\left\{\begin{array}{l}
\int_{\Omega} f(z) d z=1 \\
\int_{\Omega} z f(z) d z=\mu_{Z, 1} \\
\int_{\Omega}\left(z-\mu_{Z, 1}\right)^{j} f(z) d z=\mu_{Z, j} \quad(j=2,3,4)
\end{array}\right.
\end{gathered}
$$

We obtain the optimal PDF $f(z)$ by using the Lagrange multipliers method, with the Lagrange function given by

$$
L=H(f(z))-\left(\lambda_{0}-1\right) \int_{\Omega} f(z) d z-\lambda_{1} \int_{\Omega} z \cdot f(z) d z-\sum_{j=2}^{4} \lambda_{j} \int_{\Omega}\left(z-\mu_{Z, 1}\right)^{j} \cdot f(z) d z
$$

where $\lambda_{0}, \lambda_{1}, \ldots, \lambda_{4}$ are five Lagrange multipliers relating to the five constraints of (18).

To get the maximum entropy, we differentiate $L$ concerning $f(z)$ and then set the resulting expression equal to zero as follows:

$$
\frac{\partial L}{\partial f(z)}=0 \rightarrow-(\ln (f(z))+1)-\left(\lambda_{0}-1\right)-\lambda_{1} z-\sum_{j=2}^{4} \lambda_{j}\left(z-\mu_{z, 1}\right)^{j}=0
$$

Equation (21) yields the optimal $\operatorname{PDF} f(z)$ as it follows: 


\section{Declaration of interests}

\The authors declare that they have no known competing financial interests or personal relationships that could have appeared to influence the work reported in this paper.

$\square$ The authors declare the following financial interests/personal relationships which may be considered as potential competing interests: 